\title{
MS094.003
}

\section{Spotiton: A new method for vitrifying samples for cryoEM}

Venkata Prasad Dandey ${ }^{1}$, Hui Wei ${ }^{1}$, Zhening Zhang ${ }^{1}$, Edward T. Eng ${ }^{2}$, Alex Noble ${ }^{1}$, William J. Rice ${ }^{2}$, Ashleigh Raczkowski ${ }^{2}$, Bridget Carragher $^{1}$, Clinton S. Potter ${ }^{1}$

${ }^{1}$ The National Resource For Automated Molecular Microscopy, New York, United States, ${ }^{2}$ Simons Electron Microscopy Center, New York Structural Biology Center, New York, United States

E-mail: vdandey@nysbc.org

Almost every aspect of cryo-electron microscopy (cryoEM) has been automated over the last few decades. One of the challenges that remain to be addressed is the robust and reliable preparation of vitrified specimens[2] of suitable ice thickness. We will present results from the next generation of a new device for preparing vitrified samples. The device combines a picoliter dispensing system with a new "self-blotting"[3] grid that we have developed to provide a method for spreading a sample to a thin film without the use of externally applied filter paper. This new method[1] consumes very small amounts of protein material and results in large areas of vitrified ice of a well-defined thickness and single particles that are evenly and well distributed within the ice. We will discuss the current state of the development of Spotiton and future possibilities for further improvements. We also show here the high-resolution structure of Proteasome at 2.9 Angstrom achieved in 2days from making grid to structure with only $8 \mathrm{hr}$ of data collection on Spotiton grid.

[1] Razinkov, I et al. (2016) J. Struct. Biology,195, 190-198

[2] Dubochet, J et al. (1988) Q. Rev. Biophys. 21, 129-228.

[3] Zhang, $\mathrm{F}$ et al. (2013). Adv.Mater. 25, 4192-4198.

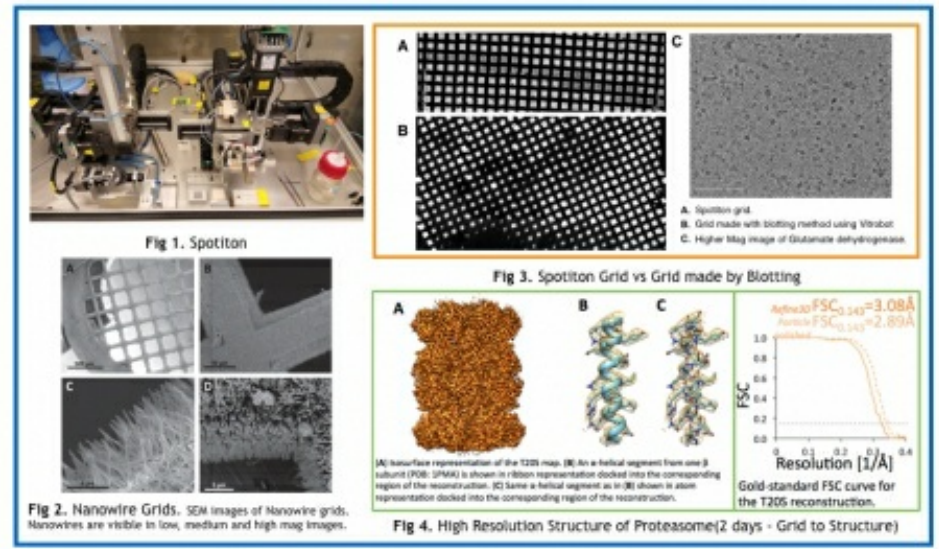

Keywords: Nano Wire grids, Self-Blotting, Cryo Electron Microscopy 